\title{
Noise removal and edge detection in ultrasound images by the topological gradient method
}

\author{
Hamdi Houichet, Maher Moakher and Badreddine Rjaibi \\ University of Tunis El Manar, National Engineering School at Tunis, Laboratory for Mathematical and Numerical Modeling in \\ Engineering Science, B.P. 37, 1002 Tunis-Belvédère, Tunisia
}

Received: 6 December 2018, Accepted: 22 November 2019

Published online: 26 December 2019.

\begin{abstract}
This paper presents a new approach for the restoration and edge detection in ultrasound images corrupted with Rayleigh-distributed multiplicative noise (speckle). We consider a semilinear PDE from which we derive the asymptotic expansion of the associated Dirichlet energy functional. We give a theoretical expansion of the topological sensitivity approach to detect and preserve the edge set in the ultrasound image during the restoration process. For the numerical solution, we design a fully-discrete forward Euler-Galerkin stepping algorithm and we present some results which illustrate the efficiency of the proposed approach.
\end{abstract}

Keywords: Speckle noise, multiplicative noise, ultrasound image, image restoration, edge detection, topological gradient.

\section{Introduction}

The restoration of images, such as active Radar, coherent Synthetic Aperture Radar (SAR), or medical images, is a challenging task in the processing and the understanding of images. Various approaches using different frameworks were proposed to deal with this kind of problem. These approaches differ by the mathematical tools used to model the problem and the type of noise that contaminates the image (additive, multiplicative). Various types of PDEs (linear, quasilinear or nonlinear) were suggested in the image restoration problem, especially, in the case of additive noise. However, the multiplicative noise model has not yet been studied thoroughly using PDEs. As far as we know, the first effective approach treating the case of Gaussian multiplicative noise, with known mean and variance was proposed by Rudin, Lions and Osher (RLO) [26]. Over the years, several variational approaches have been proposed to the multiplicative noise, also called "speckle", removal problem such as the Aubert-Aujol model (AA) [3] for SAR images. They considered a minimization problem which is derived from a maximum a posteriori (MAP) regularization approach under the assumption that the noise follows a Gamma law with mean equals one. It is well known that ultrasound images are strongly corrupted by a speckle noise [24]. For these images, the noise model reads

$$
g=u+\sqrt{u} \eta,
$$

where $g: \Omega \rightarrow \mathbb{R}$ is the observed image, $u: \Omega \rightarrow \mathbb{R}$ is the original image and $\eta: \Omega \rightarrow \mathbb{R}$ is the multiplicative noise which is a positive function and follows the Rayleigh distribution (see e.g., $[20,21,24]$ ), with $\Omega$ is an open subset in $\mathbb{R}^{2}$.

Conventional filtering techniques that operate on additive noises do not apply directly to the multiplicative noise. Many authors have made contributions to improve speckle filtering techniques like the Kuan [10,22] and Frost [15,23] filters. The major challenge when we deal with such images is how to choose and design a good model which can selectively smooth a noisy image without losing significant features such as edges. In [21], the authors proposed a speckle denoising 
model based on (1) by solving the following minimization problem

$$
\min _{\{u>0, u \in B V(\Omega)\}}\left\{\lambda T V(u)+\int_{\Omega} \frac{(g-u)^{2}}{u} \mathrm{~d} x\right\},
$$

where $B V(\Omega)$ is the space of functions with bounded variation. The first term $T V(u):=\int_{\Omega}|D u| \mathrm{d} x$ is the total variation regularization term. The second one is the data fitting term and the parameter $\lambda$ is a positive weight, which represents the trade-off between regularization and data fitting terms.

In order to preserve the edges of the image the Total Variation (TV) shows a low level of smoothing which has stair-casing effect in the homogeneous parts of the image. Motivated by the denoising model (2), to remove the speckle noise from an ultrasonic images, we here propose to study the following variational denoising model

$$
\min _{\left\{u>0, u \in H^{1}(\Omega)\right\}}\left\{\frac{\lambda}{2} \int_{\Omega}|\nabla u|^{2} \mathrm{~d} x+\int_{\Omega} \frac{(g-u)^{2}}{u} \mathrm{~d} x\right\},
$$

where $\lambda$ is a positive constant.

To increase the effect of smoothness in the homogeneous parts and to improve the quality of the restored image, we here use the square of the $H^{1}$-seminorm instead of the total variation. The main disadvantage of the $H^{1}$-seminorm is the high level of smoothing but we can control it by the topological gradient method which is the main idea to identify the edges and preserve them during the restoration process.

This paper is organized as follows. In Section 2, we present the mathematical formulation of the image restoration problem for ultrasound images, for which we show that the proposed nonlinear problem admits a unique solution in $H^{1}(\Omega)$ and satisfies a min/max principal. Then, Section 3 is devoted to computing the topological gradient by the generalized adjoint method in the case of a perforated domain by a small ellipse, which includes the perforated domain by a ball or a crack as special cases. We report the results of many numerical experiments in Section 4 , in order to discuss the behavior and the efficiency of our algorithm and to validate the theoretical results. Finally, in Section 5 some concluding remarks are given.

\section{Notations and problem formulation}

Let $\Omega$ be a bounded Lipschitz open set in $\mathbb{R}^{2}$. The minimizer $u$ of the minimization problem (3) is assumed be positive. For convenience, in the sequel we will denote by

$$
\Phi(u):=\frac{(g-u)^{2}}{u}, \quad \mathscr{J}(u):=\frac{1}{2} \int_{\Omega}|\nabla u|^{2} \mathrm{~d} x, \quad E(u):=\lambda \mathscr{J}(u)+\int_{\Omega} \Phi(u) \mathrm{d} x .
$$

Then, equation (3) can be written as

$$
\min _{u>0, u \in H^{1}(\Omega)}\left\{E(u):=\lambda \mathscr{J}(u)+\int_{\Omega} \Phi(u) \mathrm{d} x\right\} .
$$

The well-posedness of the minimization problem (5) is established in the following theorem.

Theorem 1. Let $g>0$ be in $L^{2}(\Omega)$ with $\inf _{\Omega} g>0$, then the problem (5) has a unique minimizer $u$ in $H^{1}(\Omega)$ satisfying

$$
\inf _{\Omega} g \leq u \leq \sup _{\Omega} g
$$


This result is proved using classical arguments such as compactness, semicontinuity and the convexity of the energy functional $E(\cdot)$. For background materials we refer the reader to e.g., [3, 12,20].

The Euler-Lagrange equation associated with the minimization problem (5) yields the boundary-value problem

$$
\begin{cases}-\operatorname{div}(\lambda \nabla u)+\left(\frac{u^{2}-g^{2}}{u^{2}}\right)=0, & \text { in } \Omega \\ \partial_{n} u=0, & \text { on } \Gamma\end{cases}
$$

where $\partial_{n}$ denotes the normal derivative and $n$ is the outward unit normal to $\Gamma=\partial \Omega$.

We first write this problem in a weak, or variational, form: we multiply the elliptic equation by a smooth function $v \in H^{1}(\Omega)$, integrate over $\Omega$, and apply Green's formula on the first left-hand side term, to obtain

$$
\left\{\begin{array}{l}
\text { Find } u \in H^{1}(\Omega) \text { such that, } \\
\langle\mathscr{F}(u), v\rangle=0, \quad \forall v \in H^{1}(\Omega)
\end{array}\right.
$$

where

$$
\langle\mathscr{F}(u), v\rangle=\lambda \int_{\Omega} \nabla u \cdot \nabla v \mathrm{~d} x+\int_{\Omega}\left(\frac{u^{2}-f^{2}}{u^{2}}\right) v \mathrm{~d} x .
$$

Here, the brackets $\langle\cdot, \cdot\rangle$ denote the duality product between $H^{-1}(\Omega)$ and $H^{1}(\Omega)$. In the sequel, for brevity we write $\mathscr{F}^{v}(u):=\langle\mathscr{F}(u), v\rangle$ and $D_{u} \Phi(u)=\frac{u^{2}-g^{2}}{u^{2}}$ the first derivative of $\Phi$ evaluated at $u$.

\section{The topological gradient approach}

\subsection{Asymptotic expansion}

The topological gradient method is a very well established method used in optimal design problem, see e.g., [1,2,8,16, 18]. In optimal design problems, one is usually interested in finding the optimal shape and its complement. In the context of image processing, the goal is to split the image domain into an edge set and its complement.

To present the basic idea of the topological gradient method, let $x_{0}$ be a point in $\Omega$ and $\omega$ a smooth open bounded subset in $\mathbb{R}^{2}$ containing the origin. For a small parameter $\rho>0$, let $\Omega_{\rho}$ be the perturbed domain obtained by making a perforation $\omega_{\rho}=x_{0}+\rho \omega$ around the point $x_{0}$, i.e., $\Omega_{\rho}=\Omega \backslash \omega_{\rho}$. Let $\mathscr{J}_{\rho}\left(u_{\rho}\right)$ where $u_{\rho}$ is a solution of a given PDE on the perturbed domain $\Omega_{\rho}$ and $\mathscr{J}_{\rho}(\cdot)$ is a cost function. Let $\mathscr{J}_{0}\left(u_{0}\right)$ where $u_{0}$ is the solution of the given PDE on the initial domain $\Omega$. The topological asymptotic expansion of $\mathscr{J}_{\rho}\left(u_{\rho}\right)$ when $\rho$ goes to zero is the following

$$
\mathscr{J}_{\rho}\left(u_{\rho}\right)-\mathscr{J}_{0}\left(u_{0}\right)=f(\rho) G\left(x_{0}\right)+o(f(\rho))
$$

In this expansion, $f(\rho)$ denotes a positive function going to zero with $\rho$ (in general, this function depends on the dimension of the space) and $G\left(x_{0}\right)$ is called the topological gradient or topological derivative at $x_{0}$. The topological gradient is usually simple to compute and is obtained using the solution of direct and adjoint problems defined on the initial domain. To minimize the criterion $j$, one has to create small holes at some points where the topological gradient $G$ is negative. It will be possible to build fast algorithms using this gradient-type information.

Specifically, if $\Omega$ is an image domain in $\mathbb{R}^{2}$, let $\Omega_{\rho}^{e}=\Omega \backslash E_{\rho}$ be the perturbed domain of $\Omega$ obtained by removing an ellipse $E_{\rho}$ of size $\rho$ centered at $x_{0}$ where $E_{\rho}=x_{0}+\rho E$ and $E$ is the ellipse with boundary defined by

$$
\partial E=\{X(\theta)=(a \cos (\theta), b \sin (\theta)), 0 \leq \theta \leq 2 \pi\},
$$


$a, b>0$ are the lengths of the semi-axes of $E$.

In order to preserve the edges of an image and smooth it elsewhere, we compute the asymptotic expansion of the following cost function

$$
\mathscr{J}_{\rho}\left(u_{\rho}\right)=\frac{1}{2} \int_{\Omega_{\rho}^{e}}\left|\nabla u_{\rho}\right|^{2} \mathrm{~d} x,
$$

where $u_{\rho}$ is a solution of the minimization problem (5). Next, we give links to a cracked domain by a flat crack and a perforated domain by a unit ball.

The corresponding perturbed problem associated to (6) is given by

$$
\begin{cases}-\operatorname{div}\left(\lambda \nabla u_{\rho}\right)+\left(\frac{u_{\rho}^{2}-g^{2}}{u_{\rho}^{2}}\right)=0, & \text { in } \Omega_{\rho}^{e} \\ \partial_{n} u_{\rho}=0, & \text { on } \Gamma, \\ \partial_{n} u_{\rho}=0, & \text { on } \partial E_{\rho} .\end{cases}
$$

We note that $\partial \Omega_{\rho}^{e}=\Gamma \cup \partial E_{\rho}$.

From Green's formula, it follows that $u_{\rho}$ satisfies

$$
\left\{\begin{array}{l}
\text { Find } u_{\rho} \in H^{1}\left(\Omega_{\rho}^{e}\right) \text { such that, } \\
\mathscr{F}_{\rho}^{v}\left(u_{\rho}\right)=0, \quad \forall v \in H^{1}\left(\Omega_{\rho}^{e}\right)
\end{array}\right.
$$

where

$$
\mathscr{F}_{\rho}^{v}\left(u_{\rho}\right)=\int_{\Omega_{\rho}^{e}} \lambda \nabla u_{\rho} \cdot \nabla v \mathrm{~d} x+\int_{\Omega_{\rho}^{e}} D_{u} \Phi\left(u_{\rho}\right) v \mathrm{~d} x .
$$

Our goal is to study the asymptotic behavior of the variation $\mathscr{J}_{\rho}\left(u_{\rho}\right)-\mathscr{J}_{0}\left(u_{0}\right)$ when $\rho$ goes to zero, in order to obtain the topological gradient $G^{e}$ of the proposed problem (10).

\subsection{Expression of the topological gradient}

In $[1,17]$ the authors insert a small ball and use the Laplace operator with Dirichlet boundary conditions for some semilinear PDEs. We refer the reader to the works [7,13] that study the topological gradient method for semilinear problems with Neumann boundary conditions leading to images processing and cardiac electrophysiology problems.

Let $u_{0}$ and $u_{\rho}$ be the solutions of problems (6) and (10), respectively. For simplicity, and without loss of generality, in our investigation we focus on the case of an ellipse $E_{\rho}=\rho E$, centered at the origin $0 \in E_{\rho}$. In order to prove the main result, we need the following lemmas.

Lemma 1. We have the following equality

$$
\mathscr{J}_{\rho}\left(u_{\rho}\right)-\mathscr{J}_{0}\left(u_{0}\right)=L_{\rho}\left(u_{\rho}-u_{0}\right)+\rho^{2} \delta J+\left(\rho^{2}\right)
$$

where

$$
\begin{aligned}
L_{\rho}\left(u_{\rho}-u_{0}\right) & =-\int_{\Omega_{\rho}^{e}} D_{u} \Phi\left(u_{0}\right)\left(u_{\rho}-u_{0}\right) \mathrm{d} x-\int_{\Omega_{\rho}^{e}} D_{u}^{2} \Phi\left(u_{0}\right)\left(u_{\rho}-u_{0}\right) u_{0} \mathrm{~d} x \\
\delta J & =\pi a b\left(\frac{u_{0}^{2}(0)-g^{2}(0)}{u_{0}^{2}(0)}\right) u_{0}(0) .
\end{aligned}
$$


Proof. Let us write the functional $\mathscr{J}_{0}\left(\right.$ resp. $\mathscr{J}_{\rho}$ ) at $u_{0}$ (resp. $u_{\rho}$ ), with the help of the variational formulation (7) (resp. variational formulation (11)) as follows

$$
\mathscr{J}_{0}\left(u_{0}\right)=-\int_{\Omega} D_{u} \Phi\left(u_{0}\right) u_{0} \mathrm{~d} x, \quad \mathscr{J}_{\rho}\left(u_{\rho}\right)=-\int_{\Omega_{\rho}^{e}} D_{u} \Phi\left(u_{\rho}\right) u_{\rho} \mathrm{d} x .
$$

This implies that

$$
\begin{aligned}
\mathscr{J}_{\rho}\left(u_{\rho}\right)-\mathscr{J}_{0}\left(u_{0}\right)= & \int_{\Omega_{\rho}^{e}} D_{u} \Phi\left(u_{0}\right) u_{0} \mathrm{~d} x-\int_{\Omega_{\rho}^{e}} D_{u} \Phi\left(u_{\rho}\right) u_{\rho} \mathrm{d} x+\int_{E_{\rho}} D_{u} \Phi\left(u_{0}\right) u_{0} \mathrm{~d} x \\
= & -\int_{\Omega_{\rho}^{e}} D_{u} \Phi\left(u_{0}\right)\left(u_{\rho}-u_{0}\right) \mathrm{d} x-\int_{\Omega_{\rho}^{e}}\left(D_{u} \Phi\left(u_{\rho}\right)-D_{u} \Phi\left(u_{0}\right)\right) u_{0} \mathrm{~d} x \\
& +\int_{E_{\rho}} D_{u} \Phi\left(u_{0}\right) u_{0} \mathrm{~d} x-\int_{\Omega_{\rho}^{e}}\left(D_{u} \Phi\left(u_{\rho}\right)-D_{u} \Phi\left(u_{0}\right)\right)\left(u_{\rho}-u_{0}\right) \mathrm{d} x
\end{aligned}
$$

On the other hand, since $\Phi(u)$ is $C^{3}$ and using Taylor's expansion we have

$$
\left(D_{u} \Phi\left(u_{\rho}\right)-D_{u} \Phi\left(u_{0}\right)\right) u_{0}=D_{u}^{2} \Phi\left(u_{0}\right)\left(u_{\rho}-u_{0}\right) u_{0}+\frac{1}{2} D_{u}^{3} \Phi\left(\tilde{u}_{\zeta_{\rho}^{1}}\right)\left(u_{\rho}-u_{0}\right)^{2} u_{0}
$$

and

$$
\left(D_{u} \Phi\left(u_{\rho}\right)-D_{u} \Phi\left(u_{0}\right)\right)\left(u_{\rho}-u_{0}\right) u_{0}=D_{u}^{2} \Phi\left(\tilde{u}_{\zeta_{\rho}^{2}}\right)\left(u_{\rho}-u_{0}\right)^{2} u_{0}
$$

where

$$
\tilde{u}_{\zeta_{\rho}^{i}}=\theta_{i} u_{0}+\left(1-\theta_{i}\right) u_{\rho}, 0 \leq \theta_{i} \leq 1, \text { for } i=1,2 .
$$

From which we deduce the variation of the cost function

$$
\mathscr{J}_{\rho}\left(u_{\rho}\right)-\mathscr{J}_{0}\left(u_{0}\right)=L_{\rho}\left(u_{\rho}-u_{0}\right)+j_{1}(\rho)+\mathscr{E}_{1}(\rho),
$$

where

$$
\begin{aligned}
L_{\rho}\left(u_{\rho}-u_{0}\right) & =-\int_{\Omega_{\rho}^{e}} D_{u} \Phi\left(u_{0}\right)\left(u_{\rho}-u_{0}\right) \mathrm{d} x-\int_{\Omega_{\rho}^{e}} D_{u}^{2} \Phi\left(u_{0}\right)\left(u_{\rho}-u_{0}\right) u_{0} \mathrm{~d} x, \\
j_{1}(\rho) & =\int_{E_{\rho}} D_{u} \Phi\left(u_{0}(x)\right) u_{0}(x) \mathrm{d} x, \\
\mathscr{E}_{1}(\rho) & =-\frac{1}{2} \int_{\Omega_{\rho}^{e}}\left[D_{u}^{3} \Phi\left(\tilde{u}_{\zeta_{\rho}^{1}}\right)\left(u_{\rho}-u_{0}\right)^{2} u_{0}+D_{u}^{2} \Phi\left(\tilde{u}_{\zeta_{\rho}^{2}}\right)\left(u_{\rho}-u_{0}\right)^{2} u_{0}\right] \mathrm{d} x .
\end{aligned}
$$

By using the change of variables $x=\rho y$ and applying Taylor's expansion in (15), we get $j_{1}(\rho)=\rho^{2} \delta J+o\left(\rho^{2}\right)$, where

$$
\delta J=\pi a b D_{u} \Phi\left(u_{0}(0)\right) u_{0}(0)
$$

The use of Lemma A.3 in (16) yields $\mathscr{E}_{1}(\rho)=o\left(\rho^{2}\right)$, which implies the announced result.

According to Taylor's expansion of $\mathscr{F}_{\rho}^{v \rho}$, we have

$$
\begin{aligned}
\mathscr{F}_{\rho}^{v_{\rho}}\left(u_{\rho}\right)-\mathscr{F}_{\rho}^{v_{\rho}}\left(u_{0}\right) & =D_{u} \mathscr{F}_{\rho}^{v_{\rho}}\left(u_{0}\right)\left(u_{\rho}-u_{0}\right)+\frac{1}{2} D_{u}^{2} \mathscr{F}_{\rho}^{v_{\rho}}\left(\tilde{u}_{\zeta_{\rho}}\right)\left(u_{\rho}-u_{0}\right)^{2} \\
& =D_{u} \mathscr{F}_{\rho}^{v_{\rho}}\left(u_{0}\right)\left(u_{\rho}-u_{0}\right)+\mathscr{E}_{2}(\rho),
\end{aligned}
$$


where

$$
\begin{aligned}
D_{u} \mathscr{F}_{\rho}^{v \rho}\left(u_{0}\right) \varphi & =\lambda \int_{\Omega_{\rho}^{e}} \nabla \varphi \nabla v_{\rho} \mathrm{d} x+\int_{\Omega_{\rho}^{e}} D_{u}^{2} \Phi\left(u_{0}\right) v_{\rho} \varphi \mathrm{d} x, \quad \forall \varphi \in H^{1}\left(\Omega_{\rho}^{e}\right), \\
\mathscr{E}_{2}(\rho) & =\frac{1}{2} D_{u}^{2} \mathscr{F}_{\rho}^{v_{\rho}}\left(\tilde{u}_{\zeta_{\rho}}\right)\left(u_{\rho}-u_{0}\right)^{2}=\frac{1}{2} \int_{\Omega_{\rho}^{e}} D_{u}^{3} \Phi\left(\tilde{u}_{\zeta_{\rho}}\right)\left(u_{\rho}-u_{0}\right)^{2} v_{\rho} \mathrm{d} x,
\end{aligned}
$$

For $\varphi, \phi \in H^{1}\left(\Omega_{\rho}\right)$, we introduce the Lagrangian associated to problem (11)

$$
\mathscr{L}(\varphi, \phi):=\mathscr{J}_{\rho}(\varphi)+\mathscr{F}_{\rho}^{\phi}(\varphi)
$$

The adjoint state equation is obtained by differentiating $\mathscr{L}$ with respect to $\varphi$ evaluated at $\varphi=u_{0}$ and $\phi=v_{\rho}$,

$$
D_{\varphi} \mathscr{L}(\varphi, \phi)=0, \quad \forall \varphi \in H^{1}(\Omega)
$$

or, equivalently,

$$
D_{u} \mathscr{F}_{\rho}^{v \rho}\left(u_{0}\right)(\varphi)=-L_{\rho}(\varphi), \quad \forall \varphi \in H^{1}(\Omega)
$$

By using Green's formula, we deduce the corresponding strong form of (18)

$$
\begin{cases}-\operatorname{div}\left(\lambda \nabla v_{\rho}\right)+D_{u}^{2} \Phi\left(u_{0}\right) v_{\rho}=D_{u} \Phi\left(u_{0}\right)+D_{u}^{2} \Phi\left(u_{0}\right) u_{0}, & \text { in } \Omega_{\rho}^{e} \\ \partial_{n} v_{\rho}=0, & \text { on } \Gamma \\ \partial_{n} v_{\rho}=0, & \text { on } \partial E_{\rho}\end{cases}
$$

Lemma 2. Let $v_{\rho}$ be the solution of the adjoint equation (19) and $v_{0}$ be the solution of (19) when $\rho=0$, then we have

$$
\mathscr{F}_{\rho}^{v_{\rho}}\left(u_{0}\right)-\mathscr{F}_{\rho}^{v \rho}\left(u_{\rho}\right)=-\int_{\partial E_{\rho}} \frac{\partial u_{0}}{\partial n} v_{0} d \sigma(x)-\int_{\partial E_{\rho}} \frac{\partial u_{0}}{\partial n} w_{\rho} d \sigma(x),
$$

where $w_{\rho}=v_{\rho}-v_{0}$.

Proof. The proof of this lemma is fairly standard: it is enough to apply (11), use the Green's formula and split $v_{\rho}$ as $v_{\rho}=w_{\rho}+v_{0}$.

Let us now introduce the auxiliary exterior-domain problem

$$
\begin{cases}-\Delta \phi(x)=0, & x \in \mathbb{R}^{2} \backslash E \\ \nabla \phi(x) \cdot n=\Lambda, & x \in \partial E \\ \lim _{|x| \rightarrow \infty}|\phi(x)|=0, & \end{cases}
$$

where $\Lambda \in H^{-1 / 2}(\partial E)$.

Theorem 2. $[9,11,25]$

(1) The solution $\phi$ of the exterior-domain problem (21) can be written with the help of the single-layer potential

$$
\phi(x)=\int_{\partial E} \Upsilon(x-y) \eta(y) \mathrm{d} \sigma(y)
$$

where $\Upsilon$ is the fundamental solution of the 2D Laplace operator, which is given by

$$
\Upsilon(x-y)=\frac{1}{2 \pi} \ln \left(\left(x_{1}-y_{1}\right)^{2}+\left(x_{2}-y_{2}\right)^{2}\right) .
$$


(2) There exists $\Psi \in H_{0}^{-\frac{1}{2}}(\partial E)$ solution of the boundary integral equation

$$
\frac{\Psi(x)}{2}+\int_{\partial E} \Psi(y) \nabla \Upsilon(y-x) \cdot n_{y} \mathrm{~d} \sigma(y)=\Lambda, \quad \forall y \in \partial E .
$$

(3) The jump conditions across $\partial E$ can be written as

$$
\nabla \phi \cdot n-\nabla l^{\phi} \cdot n=-\Psi, \text { and } \phi-l^{\phi}=0, \text { on } \partial E,
$$

where $l^{\phi}$ is the solution of the boundary-value problem

$$
\begin{cases}-\Delta l^{\phi}=0, & \text { in } E \\ l^{\phi}=\phi, & \text { on } \partial E\end{cases}
$$

We recall that $E$ is the ellipse with boundary defined by (8). Let $y=X(\theta)$ and $x=X(\varphi)$ be two points on $\partial E$. Then,

$$
\begin{aligned}
\int_{\partial E} \Psi(y) \nabla \Upsilon(y-x) \cdot n_{y} \mathrm{~d} \sigma(y) & =\frac{1}{2 \pi} \int_{\partial E} \frac{\left\langle y-x, n_{y}\right\rangle}{|x-y|^{2}} \Psi(y) \mathrm{d} \sigma(y), \\
& =\frac{a b}{4 \pi} \int_{0}^{2 \pi} \frac{\Psi(X(\theta))}{b^{2} \cos ^{2}\left(\frac{\theta+\varphi}{2}\right)+a^{2} \sin ^{2}\left(\frac{\theta+\varphi}{2}\right)} \mathrm{d} \theta .
\end{aligned}
$$

Note that

$$
\int_{0}^{2 \pi} \frac{\cos (2 t)}{b^{2} \cos ^{2}(t)+a^{2} \sin ^{2}(t)} \mathrm{d} t=\pi \frac{a-b}{a b(a+b)}, \quad \int_{0}^{2 \pi} \frac{\sin (2 t)}{b^{2} \cos ^{2}(t)+a^{2} \sin ^{2}(t)} \mathrm{d} t=0 .
$$

Thus, if we take $\Psi(X(\theta))=\alpha \cos (\theta)+\beta \sin (\theta)$ and $g=-\nabla v_{0}(0) \cdot n$, using (22), we obtain

$$
\alpha=-\frac{a+b}{a} \partial_{x} v(0), \quad \beta=-\frac{a+b}{b} \partial_{y} v(0) .
$$

Hence, we have

$$
\int_{\partial E} x \Psi(x) \mathrm{d} \sigma(x)=-(a+b) \pi \mathscr{D} \nabla v_{0}(0),
$$

where $\mathscr{D}=\operatorname{diag}(b, a)$. The main result is stated in the following theorem.

Theorem 3. Let $u_{0}, v_{0}$ be of class $C^{1}$ in the vicinity of the boundary of the ellipse, we have the following asymptotic expansion

$$
\mathscr{J}_{\rho}\left(u_{\rho}\right)-\mathscr{J}_{0}\left(u_{0}\right)=\rho^{2} G^{e}\left(x_{0}\right)+o\left(\rho^{2}\right),
$$

where

$$
G^{e}\left(x_{0}\right)=-\pi\left(a b\left(\frac{u_{0}^{2}\left(x_{0}\right)-g^{2}\left(x_{0}\right)}{u_{0}^{2}\left(x_{0}\right)}\right)\left(v_{0}\left(x_{0}\right)-u_{0}\left(x_{0}\right)\right)+\lambda(a+b) \nabla u_{0}\left(x_{0}\right) \cdot \mathscr{P} \nabla v_{0}\left(x_{0}\right)\right)
$$

and

$$
\mathscr{P}=\left[\begin{array}{c}
b \cos ^{2} \varphi+a \sin ^{2} \varphi(a-b) \cos \varphi \sin \varphi \\
(a-b) \cos \varphi \sin \varphi b \sin ^{2} \varphi+a \cos ^{2} \varphi
\end{array}\right],
$$

for all $\varphi \in[0, \pi]$.

Proof. For all $\left.w_{\rho}\right|_{\partial E_{\rho}} \in H^{\frac{1}{2}}\left(\partial E_{\rho}\right)$, we introduce the following extension $l_{\rho}^{w_{\rho}}$ of $w_{\rho}$ on $E_{\rho}$ which satisfies the boundary-value problem (23). 
Applying Green's formula twice on (20), from (12) and (18), we obtain

$$
\mathscr{J}_{\rho}\left(u_{\rho}\right)-\mathscr{J}_{0}\left(u_{0}\right)=\rho^{2} \delta J+j_{2}(\rho)+j_{3}(\rho)+\sum_{i=2}^{4} \mathscr{E}_{i}(\rho),
$$

where

$$
\begin{aligned}
& j_{2}(\rho)=-\int_{E_{\rho}} D_{u} \Phi\left(u_{0}\right) v_{0} \mathrm{~d} x \\
& j_{3}(\rho)=-\int_{\partial E_{\rho}}\left(u_{0}-u_{0}(0)\right)\left(\frac{\partial v_{0}}{\partial n}+\frac{\partial l_{\rho}^{w_{\rho}}}{\partial n}\right) \mathrm{d} \sigma(x) \\
& \mathscr{E}_{3}(\rho)=\lambda \int_{E_{\rho}}\left(u_{0}-u_{0}(0)\right) \Delta v_{0} \mathrm{~d} x \\
& \mathscr{E}_{4}(\rho)=-\int_{E_{\rho}} D_{u} \Phi\left(u_{0}\right) l_{\rho}^{w_{\rho}} \mathrm{d} x .
\end{aligned}
$$

To finish the proof of main result we need to prove the following lemma.

Lemma 3. For sufficiently small $\rho \geq 0$, we have the following estimations

$$
\begin{aligned}
& j_{2}(\rho)=-\rho^{2} \pi a b\left(\frac{u_{0}^{2}(0)-g^{2}(0)}{u_{0}^{2}(0)}\right) v_{0}(0)+o\left(\rho^{2}\right), \\
& j_{3}(\rho)=-\rho^{2} \pi(a+b) \nabla u_{0}\left(x_{0}\right) \cdot \mathscr{D} \nabla v_{0}\left(x_{0}\right)+o\left(\rho^{2}\right),
\end{aligned}
$$

where $\mathscr{D}=\operatorname{diag}(b, a)$.

Proof. The first estimation follows directly by the change of variables $x=\rho y$ and Taylor's expansion. For the second one, we split the variation of the adjoint state $w_{\rho}:=v_{\rho}-v_{0}$ into

$$
v_{\rho}-v_{0}=h_{\rho}+e_{\rho}
$$

where $h_{\rho}$ and $e_{\rho}$ are the solutions of the following problems, respectively

$$
\begin{cases}-\Delta h_{\rho}=0, & \text { in } \mathbb{R}^{2} \backslash E_{\rho} \\ h_{\rho} \rightarrow 0, & \text { at } \infty \\ \nabla h_{\rho}=-\nabla v_{0}(0) \cdot n, & \text { on } \partial E_{\rho}\end{cases}
$$

and

$$
\begin{cases}-\operatorname{div}\left(\lambda \nabla e_{\rho}\right)+D_{u}^{2} \Phi\left(u_{0}\right) v_{\rho}-D_{u}^{2} \Phi\left(u_{0}\right) v_{0}=0, & \text { in } \Omega_{\rho}^{e} \\ \nabla e_{\rho} \cdot n=-\nabla h_{\rho} \cdot n, & \text { on } \Gamma, \\ \nabla e_{\rho} \cdot n=\nabla v_{0}(0) \cdot n-\nabla v_{0} \cdot n, & \text { on } \partial E_{\rho}\end{cases}
$$


Then, $j_{3}$ becomes

$$
\begin{aligned}
j_{3}(\rho) & =-\int_{\partial E_{\rho}}\left(u_{0}-u_{0}(0)\right)\left(\nabla v_{0} \cdot n+\nabla l_{\rho}^{h_{\rho}} \cdot n\right) \mathrm{d} \sigma(x)+\mathscr{E}_{5}(\rho), \\
& =\int_{\partial E_{\rho}}\left(u_{0}-u_{0}(0)\right) \Psi\left(\frac{x}{\rho}\right) \mathrm{d} \sigma(x)+\sum_{i=5}^{6} \mathscr{E}_{i}(\rho), \\
& =\rho \int_{\partial E}\left(u_{0}(\rho x)-u_{0}(0)+\rho x \nabla u_{0}(0)-\rho x \nabla u_{0}(0)\right) \Psi(x) \mathrm{d} \sigma(x)+\sum_{i=5}^{6} \mathscr{E}_{i}(\rho), \\
& =\rho^{2} \nabla u_{0}(0) \int_{\partial E} x \Psi(x) \mathrm{d} \sigma(x)+\sum_{i=5}^{7} \mathscr{E}_{i}(\rho),
\end{aligned}
$$

where

$$
\begin{aligned}
& \mathscr{E}_{5}(\rho)=-\int_{\partial E_{\rho}}\left(u_{0}-u_{0}(0)\right)\left(\nabla l_{\rho}^{w_{\rho}} \cdot n-\nabla l_{\rho}^{h_{\rho}} \cdot n\right) \mathrm{d} \sigma(x), \\
& \mathscr{E}_{6}(\rho)=-\int_{\partial E_{\rho}}\left(u_{0}-u_{0}(0)\right)\left(\partial_{n} v_{0}-\nabla v_{0}(0) \cdot n\right) \mathrm{d} \sigma(x), \\
& \mathscr{E}_{7}(\rho)=\rho \int_{\partial E} \Psi(x)\left(u_{0}(\rho x)-u_{0}(0)-\nabla u_{0}(0) \rho x\right) \mathrm{d} \sigma(x) .
\end{aligned}
$$

Using (24) and Lemma A.3, then (29) holds.

We now proceed to complete the proof of Theorem 3.

This implies

More generally, if $E^{\prime}$ is an ellipse of the form (8), there exists a rotation

$$
R_{\varphi}=\left[\begin{array}{cc}
\cos \varphi & -\sin \varphi \\
\sin \varphi & \cos \varphi
\end{array}\right]
$$

such that $E=R_{\varphi}\left(E^{\prime}\right)$. Therefore, we get

$$
\mathscr{P}=R_{\varphi}^{T} \mathscr{D} R_{\varphi}
$$

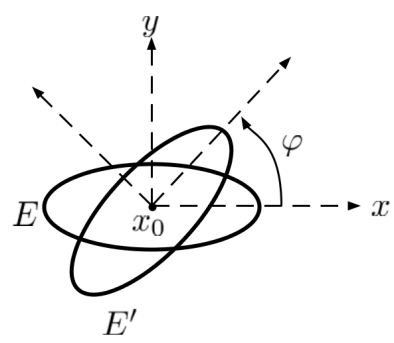

$$
j_{3}(\rho)=-\rho^{2} \pi(a+b) \nabla u_{0}\left(x_{0}\right) \cdot \mathscr{P} \nabla v_{0}\left(x_{0}\right)+o\left(\rho^{2}\right) .
$$

Let $\delta J, j_{2}$ and $j_{3}$ given by (13), (28), (35), respectively, then we obtain the topological gradient for the perforated domain by an ellipse centered at $x_{0}$

$$
G^{e}\left(x_{0}\right)=-\pi\left(a b\left(\frac{u_{0}^{2}\left(x_{0}\right)-g^{2}\left(x_{0}\right)}{u_{0}^{2}\left(x_{0}\right)}\right)\left(v_{0}\left(x_{0}\right)-u_{0}\left(x_{0}\right)\right)+\lambda(a+b) \nabla u_{0}\left(x_{0}\right) \cdot \mathscr{P} \nabla v_{0}\left(x_{0}\right)\right) .
$$

Remark. In the proof of Lemma 3, we made a change of variable into the ellipse $E$, in this case we pose $h_{\rho}(x)=\rho H\left(\frac{x}{\rho}\right)$, where $H$ is the solution of the exterior-domain problem (21) defined in $\mathbb{R}^{2} \backslash E$ with $\Lambda=-\nabla v_{0}(0) \cdot n$.

\subsection{Perforation by ellipses connection's to perforation by balls and cracks}

The results of this article generalize results obtained for the case of topological gradient for domains perforated by balls or cracks. To recover results of a perforated domain by a ball centered at $x_{0}$ it suffices to take $a=b=1$ (see [14, Chapter 
2]). So the expression of the topological gradient (3.2) in the case of a perforated domain by a ball reduces to

$$
G^{b}\left(x_{0}\right)=-\pi\left(\left(\frac{u_{0}^{2}\left(x_{0}\right)-g^{2}\left(x_{0}\right)}{u_{0}^{2}\left(x_{0}\right)}\right)\left(v_{0}\left(x_{0}\right)-u_{0}\left(x_{0}\right)\right)+2 \lambda \nabla u_{0}\left(x_{0}\right) \cdot \nabla u_{0}\left(x_{0}\right)\right) .
$$

Similarly, when we take $a=1$ and tend $b$ to zero, the expression of the topological gradient (3.2) becomes the expression of the topological gradient for a domain with a crack at the point $x_{0}$, with unit outward normal vector $n_{c}$, given by

$$
G^{c}\left(x_{0}, n_{c}\right)=-\pi \lambda \nabla u_{0}\left(x_{0}\right) \cdot n_{c} \nabla v_{0}\left(x_{0}\right) n_{c} .
$$

\section{Numerical experiments}

In this section, we discuss a fully discrete forward Euler-Galerkin method for our semilinear equation (6). As is well known, a time marching method is one of many convenient ways to solve the resulting Euler-Lagrange equations like (6). The main idea is to introduce an artificial time parameter $t$ and compute the steady-state solution of the time-dependent semilinear partial differential equation as the follows

$$
\begin{cases}\frac{\partial u_{0}}{\partial t}=-\operatorname{div}\left(\lambda \nabla u_{0}\right)+D_{u} \Phi\left(u_{0}\right), & \text { on } \Omega \times[0, T], \\ \frac{\partial u_{0}}{\partial n}=0, & \text { in } \partial \Omega \times[0, T], \\ u_{0}(., 0)=g, & \text { on } \Omega,\end{cases}
$$

where, $T>0$ denotes the final time of the evolutionary process. It is well known $[12,19,20]$ that, for $g \in L^{2}(\Omega)$ such that $\inf _{\Omega} g>0$ there exists a unique solution $u \in L^{2}\left([0, T] ; H^{1}(\Omega)\right)$ to the semilinear parabolic problem (38) satisfying

$$
\left(u_{t}, \phi\right)=(\lambda \nabla u, \nabla \phi)+\left(D_{u} \Phi(u), \phi\right), \quad \forall \phi \in H^{1}(\Omega),
$$

where $(\cdot, \cdot)$ denotes the $L^{2}(\Omega)$-inner product. In order to obtain a numerical approximation of problem (6), we will discretize the spatial and temporal variables in the weak formulation of the evolution (39) and use a finite-element method in space and a fully implicit finite-difference scheme in time.

Let $\mathscr{T}_{h}=\{T\}_{T \in \mathscr{T}_{h}}$ denote a partition of $\Omega$ into disjoint triangles $T$ such that no vertex of any triangle lies in the interior or on a side of another triangle and such that the union of the triangles determines a polygonal domain $\Omega_{h} \subset \Omega$ with boundary vertices on $\partial \Omega$. The maximum value of the diameter of all triangles $T \in \mathscr{T}_{h}$ is denoted by $h$. Based on the mesh $\mathscr{T}_{h}$, we define the finite-element space $X_{h} \subset H^{1}(\Omega)$ such that

$$
X_{h}=\left\{\phi \in C(\bar{\Omega}) ;\left.\phi\right|_{T} \in \mathbb{P}_{1}(T) \forall T \in \mathscr{T}_{h}\right\},
$$

where $\mathbb{P}_{1}$ is the standard linear space of polynomials of degree less or equal to 1.

Based on the weak formulation (39), we formulate the spatially semi-discrete problem, which for $t \geq 0$ consists in finding $u_{h}(\cdot, t) \in V_{h}=H^{1}\left(0, T ; X_{h}\right)$, such that

$$
\left(u_{h, t}, \phi_{h}\right)=\left(\lambda \nabla u_{h}, \nabla \phi_{h}\right)+\left(D_{u} \Phi\left(u_{h}\right), \phi_{h}\right), \quad \forall \phi_{h} \in X_{h}, t>0, \text { with } u_{h}(0)=g_{h},
$$

where $g_{h}$ is some approximation of $g$ in $X_{h}$. Moreover, we consider a partition of the time interval $[0, T]$ into $M$ equallyspaced sub-intervals $I_{n}=\left[t^{n-1}, t^{n}\right], n=1, \ldots, M$, defined by the following points

$$
0=t^{0}<t^{1}<\cdots<t^{M-1}<t^{M}=T,
$$


with $t^{n}=n \Delta t$ and $\Delta t=T / M$ is the time-step. We then define the fully-discrete solution to be the piecewise constant in time function, $u_{h}(x, t)$, given by

$$
u_{h}(x, t)=U^{n}(x), \forall t \in I_{n}, 1 \leq n \leq M
$$

The fully-discrete finite-element approximation to the problem (39) is defined as follows: For $n=0,1, \ldots, M$, find $U^{n} \in V_{h}$ such that

$$
\left(\bar{\partial}_{t} U^{n}, \phi_{h}\right)=\left(\lambda \nabla U^{n-1}, \nabla \phi_{h}\right)+\left(D_{u} \Phi\left(U^{n-1}\right), \phi_{h}\right), \quad \forall \phi_{h} \in X_{h},
$$

where $\bar{\partial}_{t} U^{n}=\left(U^{n}-U^{n-1}\right) / \Delta t$ is an approximation of $\partial_{t} u$ at $t=t^{n}$. Furthermore, for each $n=1, \ldots, M$, there exists a unique solution to (41). The proof is similar to that presented in $[29,31]$.

Remark. The approximation of the adjoint problem (19), which is a linear problem, immediately leads to the solution of a linear algebraic system, for which a well-posedness is guaranteed via the Lax-Milgram lemma.

\subsection{Algorithm}

Our algorithm consists in inserting small cracks in regions where the topological gradient is smaller than a given threshold $\alpha<0$ and which can be seen as edges of the image. In addition, we assume that the edges of the images can be considered as cracks $[4,6,18]$ which justifies the use of the topological gradient (37).

Let us now make precise the structure of the topological gradient. We write $G^{c}$ as $G^{c}\left(x_{0}, n_{c}\right)=\left\langle M(x) n_{c}, n_{c}\right\rangle_{E}$, where $\langle\cdot, \cdot\rangle_{E}$ is the Euclidean inner product and $M(x)$ is the $2 \times 2$ symmetric matrix defined by $[4,6,18]$

$$
M(x)=-\frac{\pi \lambda}{2}\left(\nabla u_{0}(x) \nabla v_{0}(x)^{T}+\nabla v_{0}(x) \nabla u_{0}(x)^{T}\right)
$$

where $u_{0}$ and $v_{0}$ are the solutions of the direct and adjoint state, respectively.

Remark. The direct state and the adjoint state are computed in the initial domain without cracks. For a given $x, G^{c}\left(x_{0}, n_{c}\right)$ takes its minimal value when $n_{c}$ is the eigenvector associated to the smallest eigenvalue $e_{\min }$ of $M$. This value will be considered as the topological gradient associated to the optimal orientation of the crack.

The steps of the algorithm are the following:

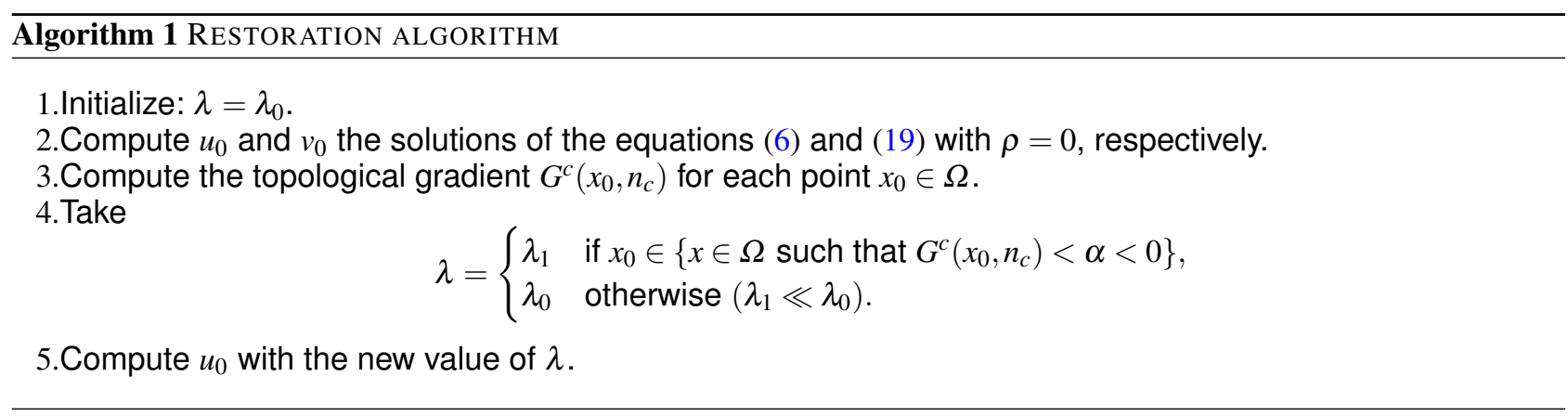

\subsection{Results}

In this work, all simulations were implemented in Matlab 8.5 (R2015a) running on a Desktop with Intel core i3 processor, CPU at $2.4 \mathrm{GHz}$ and 4GB RAM memory. In the numerical experiments, we considered gray-value images and the quality of the restored image is measured by the Signal-to-Noise Ratio (SNR), Peak Signal-to-Noise Ratio (PSNR) and the 
Structural Similarity Index Measurement (SSIM) [30]. For example, the latter allows to estimate the quality of an image, $I$, with respect to a reference image, $I_{0}$, and is defined by the following expression

$$
\operatorname{SSIM}=\frac{\left(2 \mu_{I} \mu_{I_{0}}+c_{1}\right)\left(2 \sigma_{I_{1} I_{0}}+c_{2}\right)}{\left(\mu_{I}^{2}+\mu_{I_{0}}^{2}+c_{1}\right)\left(\sigma_{I}^{2}+\sigma_{I_{0}}^{2}+c_{2}\right)}
$$

where $\mu, \sigma^{2}, \sigma$ are the mean, the variance, the covariance, respectively, and $c_{1}$ and $c_{2}$ are two positive parameters.

The performance of the proposed approach is illustrated for multiplicative speckle noise removal and edges detection. We compare our approach between the classical nonlinear filters like the Frost filter [15], the Kuan filter [22], the TV model (2) and the Laplace model, i.e., with the model (5) without using the topological gradient in order to see its importance in image denoising problem. Figure 1 shows the results obtained by the different models for a synthetic image of size $100 \times 100$ pixels which is contaminated by a speckle noise of variance $\sigma^{2}=0.02$. We show that the results obtained by PDE (see Figures 1(d), 1(e) and 1(f)) are better than the result obtained by the classical filter (see Figures 1(b) and 1(c)). We see from Figures 1(b) and 1(b) that the image is still noisy. From Figure 1(c), we can see that the Laplace model admits a strong smoothing rate and the image is blurred. The result in Figure 1(f) is very close to the original one and we can see that our method gives a good result compared with the other models. The main difference between the noisy image and restored ones is compared quantitatively in Table 1 by using the SNR and SSIM indicators.

\begin{tabular}{|l|c|c|c|c|c|c|}
\cline { 2 - 7 } \multicolumn{1}{c|}{} & Figure 1(a) & Figure 1(b) & Figure 1(c) & Figure 1(d) & Figure 1(e) & Figure 1(f) \\
\hline PSNR (dB) & 21.18 & 27.46 & 29.05 & 31.22 & 32.53 & 35.37 \\
\hline SNR $(\mathrm{dB})$ & 17.03 & 23.19 & 24.78 & 25.02 & 28.25 & 31.14 \\
\hline SSIM & 0.31 & 0.79 & 0.81 & 0.88 & 0.91 & 0.95 \\
\hline
\end{tabular}

Table 1: The main difference between the noisy image and restored ones which is compared quantitatively by using the SNR and SSIM indicators.

In order to test the effectiveness and the impact of the topological gradient in the restoration process, we zoom the result in Figure 1, we see that the image is blurred (see Figure 2) which is due to the strong level of smoothing of Laplace operator and the edges are not preserved. However, from the TV model we can see few oscillations in the homogenizes parts of the image. From our method we can see that the edges are well identified.

In Figure 3, we make a numerical comparison with the three models. We show the reconstruction error between the restored image and the original one. We can see that the distribution of the error is mainly concentrated near the main edges of the image.

In Figure 4, we display the noisy images which are used as initial condition in (7) and obtained by adding a speckle noise with different values of variance $\sigma^{2} \in\{0.04,0.09,0.3,0.6,0.9\}$ to the original image. In the second row of Figure 4, we give the main edges of the image. We show that the length of contours is different from image to another. In the first test the edges are very tiny however when the noise level increases, the length increases. Third row of Figure 4 shows the restored image using our method which is visually excellent. The quality of restored images are compared quantitatively in Figure 5 by the SSIM, PSNR and SNR indicators. In this figure, we display images which represent the main edges of images and the results show the efficiency of the proposed approach as an edges detection method which indicate that the performance of our approach is good, even for a high level of noise.

Figure 5 shows the evolution of SNR, PSNR and SSIM of the restored images as functions of the variance $\sigma^{2}$. We can see that when we increase the variance $\sigma^{2}$ of noise, the SNR and PSNR are monotonically decreasing, but, the SSIM values are still higher than 0.9 . 


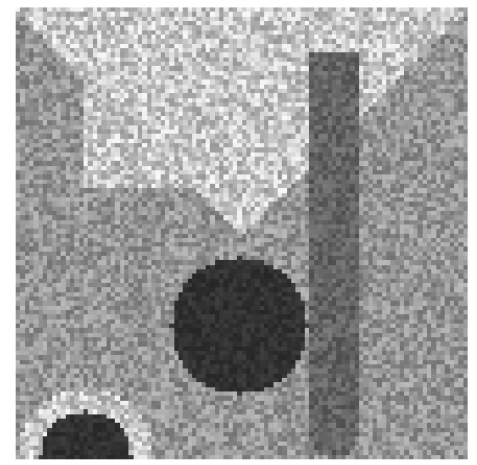

(a)

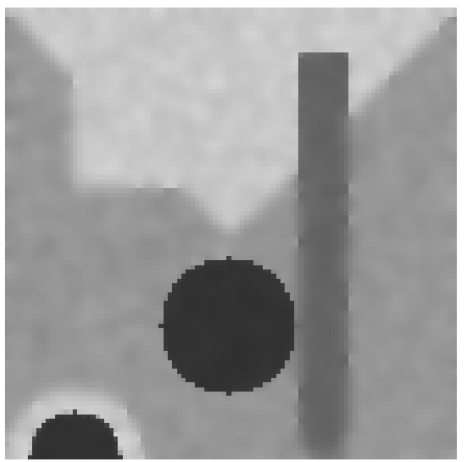

(d)

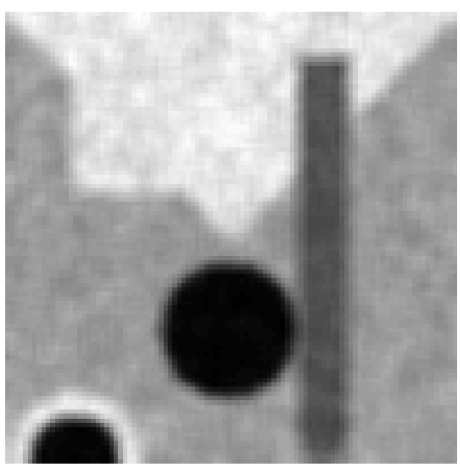

(b)

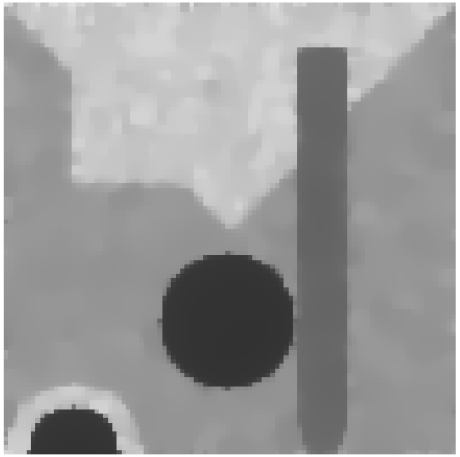

(e)

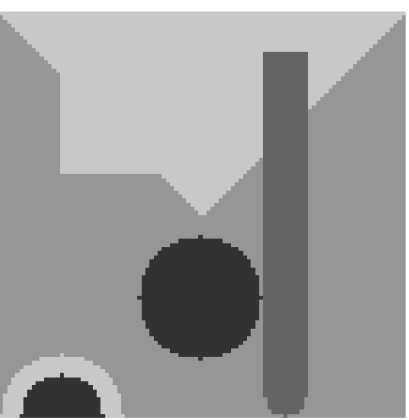

(g)

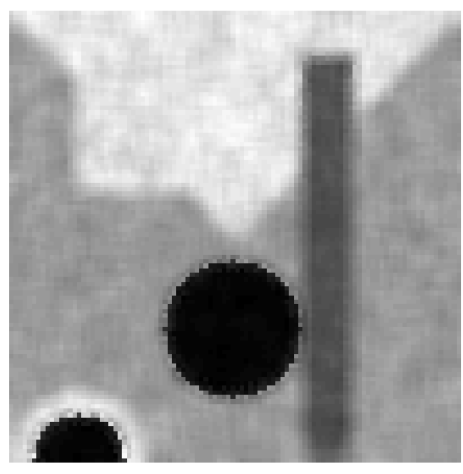

(c)

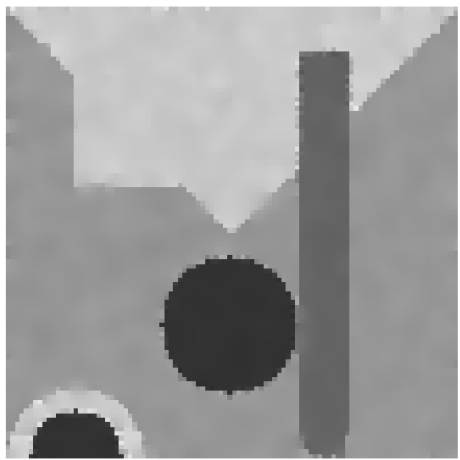

(f)

(g)

Fig. 1: From left to right and top to bottom: (a) The noisy image (PSNR=21.18dB, SSIM=0.31), (b) Frost filter (PSNR= 27.46dB, SSIM=0.79), (c) Kuan filter PSNR=29.05dB, SSIM=0.81), (d) Laplace model (PSNR= 31.22dB, SSIM= 0.88), (e) TV model (PSNR $=32.53 \mathrm{~dB}, \mathrm{SSIM}=0.91)$, (f) our approach $(\mathrm{PSNR}=35.37 \mathrm{~dB}, \mathrm{SSIM}=0.95)$ and $(\mathrm{g})$ the original image. 

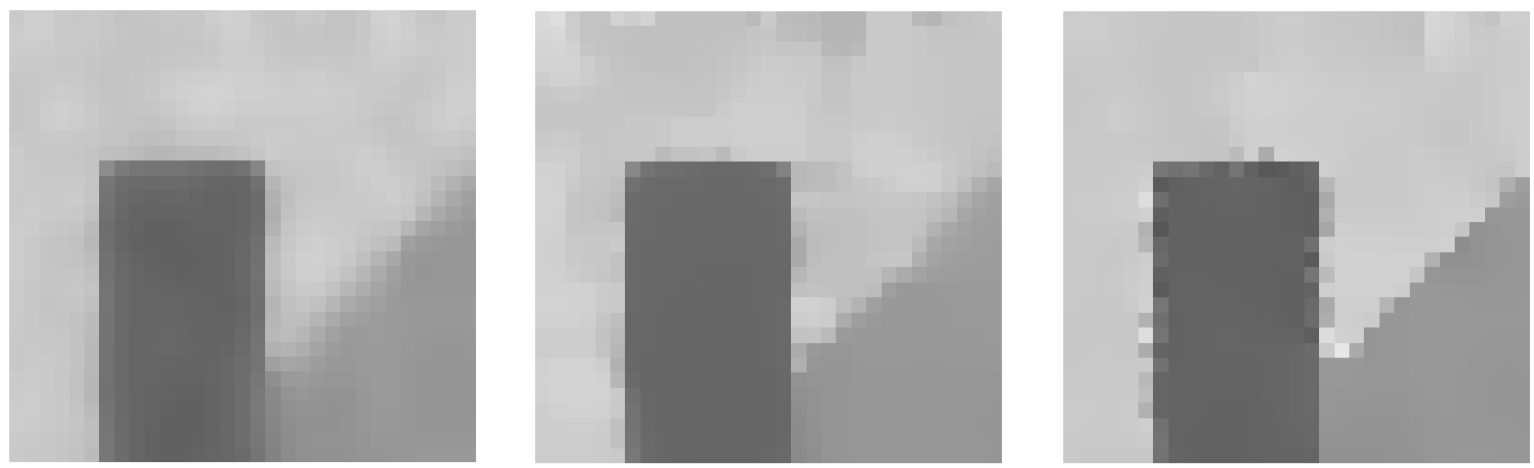

Fig. 2: From left to right: Zoom of the Laplace model, TV model and our approach.
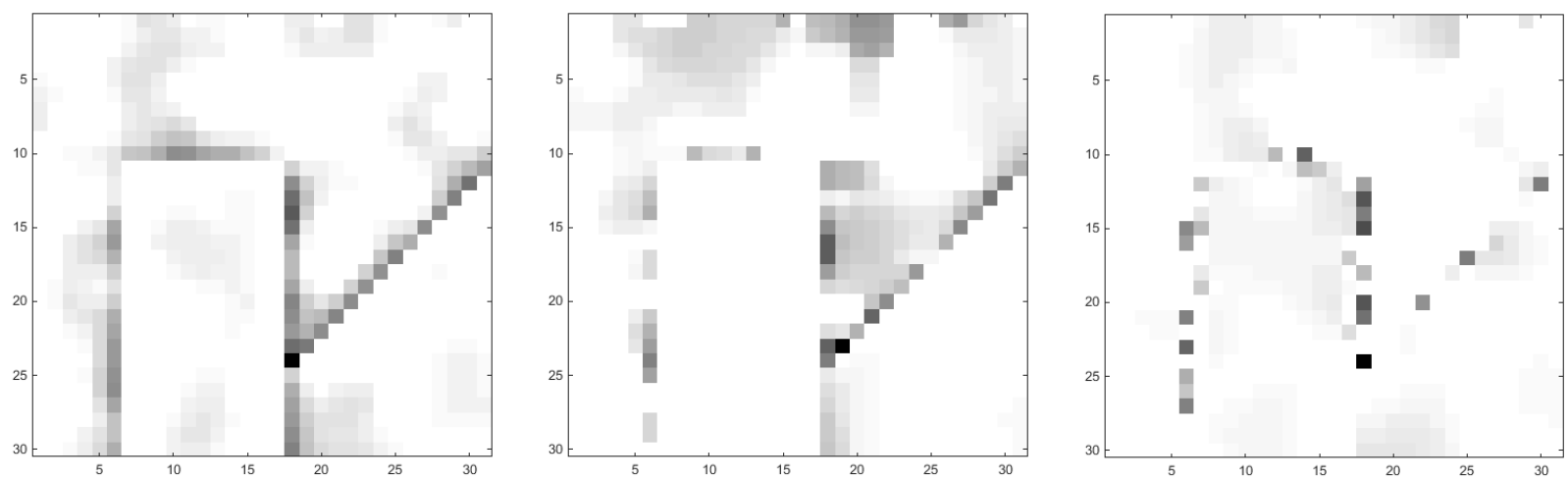

Fig. 3: From left to righ: Zoom of the reconstruction error between the restored image by the Laplace model, TV model and our approach compared with the reference image, respectively.

Figures 6-9 show the efficiency of the proposed method in real image denoising (SAR, medical, microscopic, etc). From these experiments, we can see that the proposed model gives a satisfactory restoration in smooth parts of the images and convincing identification of edges.

\section{Conclusion}

We have studied a new method based on the topological sensitivity approach to remove speckle noise in ultrasound images. We have calculated the topological gradient for a perforated domain by ellipses, cracks and balls, respectively. The edges are well preserved and the image is smoothed elsewhere during the restoration process. The obtained numerical results encourage further analysis of the proposed technique. For instance, we can consider a similar approach for a higher-order PDE in Ultrasound Computed Tomography [5,27,28]. 

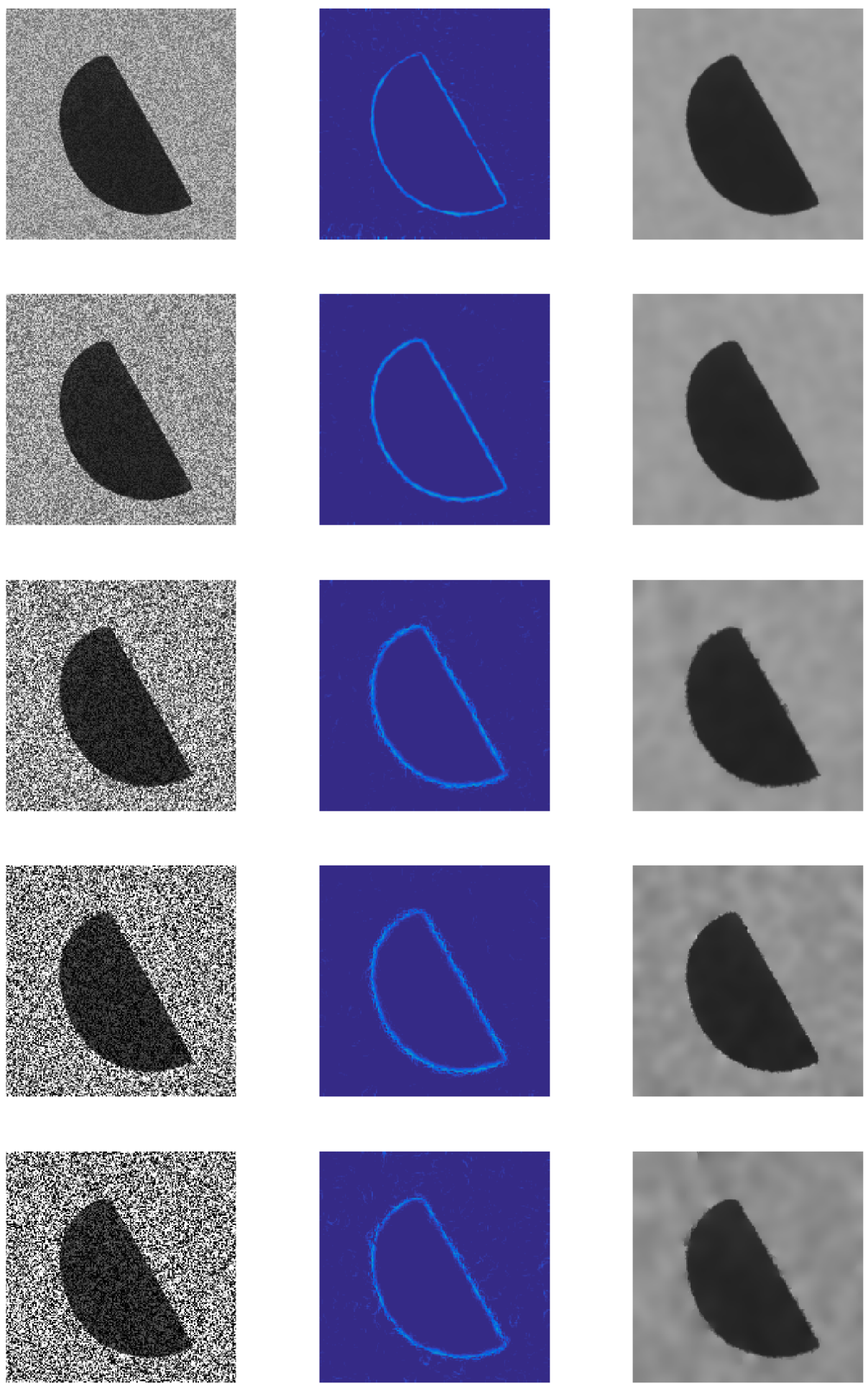

Fig. 4: From left to right and top to bottom: The noisy images of different level of noise $\left(\sigma^{2}=0.04,0.09,0.3,0.6\right.$ and $0.9)$, the edges detected by the topological gradient and the restored images. 

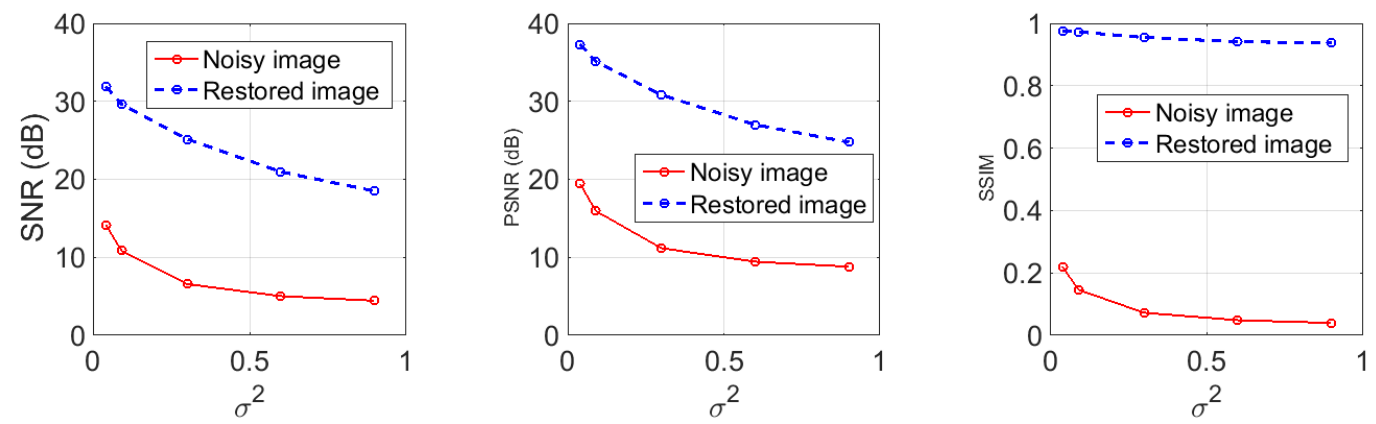

Fig. 5: Evolution of the SNR, PSNR and SSIM of the restored image by our approach as a function of level of noise $\sigma^{2}$.

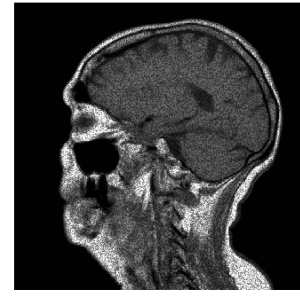

(a)

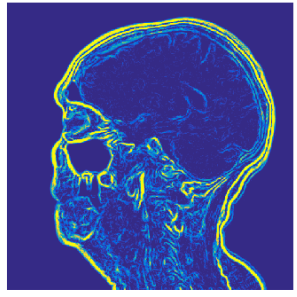

(b)

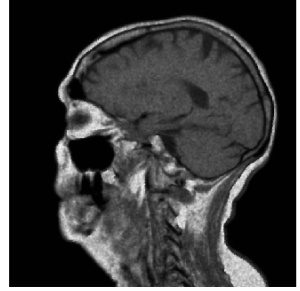

(c)

Fig. 6: (a) Noisy image ( $\mathrm{SNR}=11.61 \mathrm{~dB}, \mathrm{PSNR}=22.24 \mathrm{~dB}, \mathrm{SSIM}=0.67)$, (b) Contour and (c) Restored image ( $\mathrm{SNR}=$ $19.50 \mathrm{~dB}, \mathrm{PSNR}=30.50 \mathrm{~dB}, \mathrm{SSIM}=0.899) \sigma^{2}=0.08$.

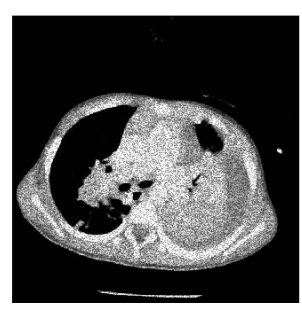

(a)

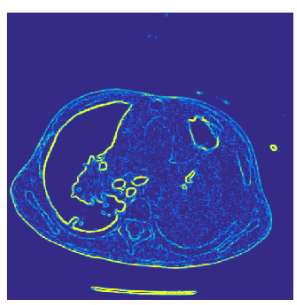

(b)

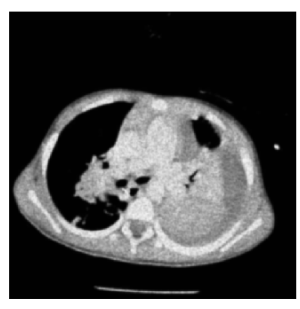

(c)

Fig. 7: (a) Noisy image $(\mathrm{SNR}=11.88 \mathrm{~dB}, \mathrm{PSNR}=19.76 \mathrm{~dB}, \mathrm{SSIM}=0.72)$, (b) Contour and (c) Restored image (SNR= $18.74 \mathrm{~dB}, \mathrm{PSNR}=26.97 \mathrm{~dB}, \mathrm{SSIM}=0.88) \sigma^{2}=0.08$.

\section{Appendix A}

Lemma A.1. Let $H$ be the solution of (21), then, the following estimates are satisfied:

$$
\begin{aligned}
& |H(x)| \leq \frac{c}{|x|}, \quad\left\|H\left(\frac{x}{\rho}\right)\right\|_{0, \Omega_{\rho}^{e}} \leq O(\rho \sqrt{-\log \rho}), \\
& |\nabla H(x)| \leq \frac{c}{|x|^{2}} \quad \text { and } \quad\left\|\nabla H\left(\frac{x}{\rho}\right)\right\|_{0, \Omega_{\rho}^{e}}=O(\rho) .
\end{aligned}
$$




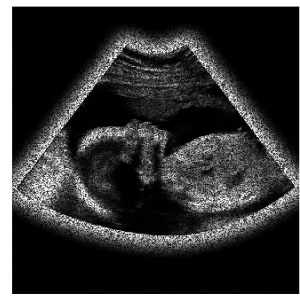

(a)

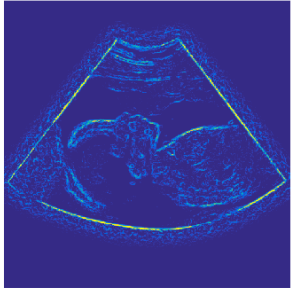

(b)

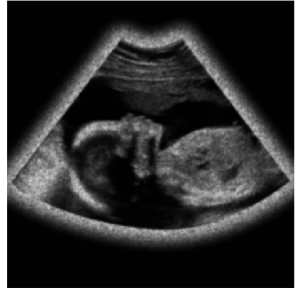

(c)

Fig. 8: (a) Noisy image $(\mathrm{SNR}=6.54 \mathrm{~dB}, \mathrm{PSNR}=17.8 \mathrm{~dB}, \mathrm{SSIM}=0.63)$, (b) Contour and (c) Restored image ( $\mathrm{SNR}=$ 16.01dB, PSNR $=28.46 \mathrm{~dB}, \mathrm{SSIM}=0.87) \sigma^{2}=0.3$.

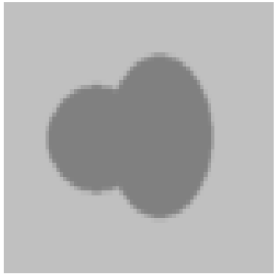

(a)

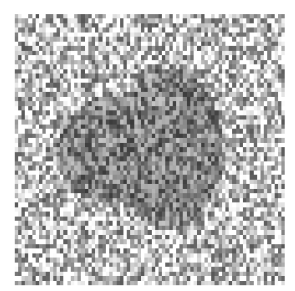

(b)

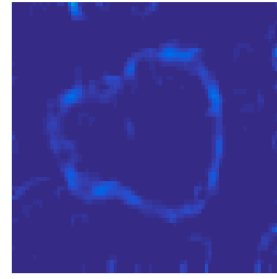

(c)

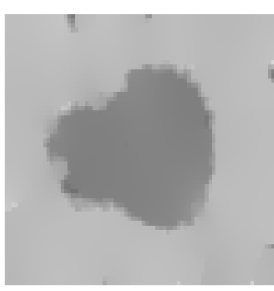

(d)

Fig. 9: (a) Original image, (b) Noisy image $(\mathrm{SNR}=10.9 \mathrm{~dB}, \mathrm{PSNR}=13.85 \mathrm{~dB}, \mathrm{SSIM}=0.067)$, (c) Contour and (d) Restored image $(\mathrm{SNR}=26.21 \mathrm{~dB}, \mathrm{PSNR}=29.66 \mathrm{~dB}, \mathrm{SSIM}=0.90) \sigma^{2}=0.1$.

Lemma A.2. Let $v_{\rho}$ and $v_{0}$ the solution of (19) with $\rho>0$ and $\rho=0$ and $e_{\rho}$ the solution of (31), then

$$
\begin{aligned}
\left\|v_{\rho}-v_{0}\right\|_{0, \Omega_{\rho}^{e}}=O\left(\rho^{2} \sqrt{-\log (\rho)}\right), & \left|v_{\rho}-v_{0}\right|_{1, \Omega_{\rho}^{e}}=O(\rho), \\
\left\|u_{\rho}-u_{0}\right\|_{0, \Omega_{\rho}^{e}}=O\left(\rho^{2} \sqrt{-\log (\rho)}\right), & \left|u_{\rho}-u_{0}\right|_{1, \Omega_{\rho}^{e}}=O(\rho) .
\end{aligned}
$$

Proof. We prove here only the first tow estimations (A.1), For the proof of the variation of the second estimations (A.2), we use the same step. we recall that in previous section, we approximate $v_{\rho}$ as $v_{\rho}=v_{0}+\rho H\left(\frac{x}{\rho}\right)+e_{\rho}$, where $e_{\rho}$ solves

$$
\begin{cases}-\operatorname{div}\left(\lambda \nabla e_{\rho}\right)+D_{u}^{2} \Phi\left(u_{0}\right) e_{\rho}=-D_{u}^{2} \Phi\left(u_{0}\right) \rho H\left(\frac{x}{\rho}\right), & \text { in } \Omega_{\rho}^{e}, \\ \nabla e_{\rho} \cdot n=-\nabla H\left(\frac{x}{\rho}\right) \cdot n, & \text { on } \Gamma, \\ \nabla e_{\rho} \cdot n=\nabla v_{0}(0) \cdot n-\nabla v_{0} \cdot n, & \text { on } \partial E_{\rho}\end{cases}
$$

We approximate again $e_{\rho}$ as $e_{\rho}=r_{\rho}+s_{\rho}$, where $r_{\rho}$ and $s_{\rho}$ are the solutions of the following problems, respectively

$$
\begin{cases}\Delta r_{\rho}=0, & \text { in } \Omega_{\rho}^{e} \\ \nabla r_{\rho} \cdot n=0, & \text { on } \Gamma \\ \nabla r_{\rho} \cdot n=\nabla v_{0}(0) \cdot n-\nabla v_{0} \cdot n, & \text { on } \partial E_{\rho}\end{cases}
$$


and

$$
\begin{cases}-\operatorname{div}\left(\lambda \nabla s_{\rho}\right)+D_{u}^{2} \Phi\left(u_{\zeta_{\rho}}\right) s_{\rho}=-D_{u}^{2} \Phi\left(u_{\zeta_{\rho}}\right)\left(\rho H\left(\frac{x}{\rho}\right)+r_{\rho}\right), & \text { in } \Omega_{\rho}^{e}, \\ \nabla s_{\rho} \cdot n=-\nabla H \cdot n, & \text { on } \Gamma, \\ \nabla s_{\rho} \cdot n=0, & \text { on } \partial E_{\rho} .\end{cases}
$$

Standard computations as [1] leads

$$
\left\|r_{\rho}\right\|_{H^{1}\left(\Omega_{\rho}^{e}\right) / \mathbb{R}} \leq c \rho^{2}, \quad\left\|s_{\rho}\right\|_{1, \Omega_{\rho}} \leq O\left(\rho^{2} \sqrt{-\log (\rho)}\right) .
$$

Applying the Cauchy-Schwartz inequality and using the above estimations, we obtain

$$
\left\|e_{\rho}\right\|_{1, \Omega_{\rho}^{e}}=O\left(\rho^{2} \sqrt{-\log (\rho)}\right) .
$$

Using Lemma A.1 and the inequality (A.3), we have

$$
\left\|v_{\rho}-v_{0}\right\|_{0, \Omega_{\rho}^{e}}=\left\|\rho H\left(\frac{x}{\rho}\right)+e_{\rho}\right\|_{0, \Omega_{\rho}^{e}}
$$

which implies the announced result.

Lemma A.3. Let $\mathscr{E}_{i}$ for $i \in \llbracket 1 \ldots 7 \rrbracket$ given by (16), (17), (26), (27) and (32)-(34), then

$$
\mathscr{E}_{i}(\rho)=o\left(\rho^{2}\right), \quad i \in \llbracket 1 \ldots 7 \rrbracket .
$$

Proof. For $\mathscr{E}_{1}(\rho), \mathscr{E}_{2}(\rho), \mathscr{E}_{6}(\rho)$ and $\mathscr{E}_{7}(\rho)$, using the Hölder inequality, the regularity of $\Phi$, applying Lemma A.2, making the change of variables $x=\rho y$ and using Taylor's expansion, yields

$$
\mathscr{E}_{i}(\rho)=o\left(\rho^{2}\right), \text { for } i \in\{1,2,6,7\}
$$

Similarly for $\mathscr{E}_{3}(\rho)$, applying the adjoint equation (19) with $\rho=0$, making the change of variables $x=\rho y$ and using Taylor's expansion, we obtain

$$
\mathscr{E}_{3}(\rho)=o\left(\rho^{2}\right)
$$

Since for $\mathscr{E}_{4}(\rho)$, using the change of variables $x=\rho y$, a trace theorem on $B \backslash E$ (where $B$ is a ball contains $E$ ), and the fact that in the space $H^{1}(\Omega) / \mathbb{R}$ the seminorm is equivalent of the norm, $\left(\|X\|_{H^{1}(\Omega) / \mathbb{R}} \leq c|X|_{H^{1}(\Omega)}\right)$, a change of variable again, the elliptic regularity and applying estimations (A.1), we get

$$
\mathscr{E}_{4}(\rho)=o\left(\rho^{2}\right) .
$$

In a similar fashion as is done for $\mathscr{E}_{4}$, we obtain

$$
\mathscr{E}_{5}(\rho)=o\left(\rho^{2}\right)
$$

\section{Acknowledgments}

This work was financially supported by the "PHC Utique" program NAMRED of the French Ministry of Foreign Affairs and Ministry of higher education, research and innovation and the Tunisian Ministry of higher education and scientific research in the CMCU project number $18 \mathrm{G} 1502$. 


\section{Competing interests}

The authors declare that they have no competing interests.

\section{Authors' contributions}

All authors have contributed to all parts of the article. All authors read and approved the final manuscript.

\section{References}

[1] S. Amstutz. Topological sensitivity analysis for some nonlinear PDE system. J. Math. Pures Appl., 85(4):540-557, 2006.

[2] S. Amstutz, I. Horchani, and M. Masmoudi. Crack detection by the topological gradient method. Control and Cybernetics, 34(1):81-101, 2005.

[3] G. Aubert and J.-F. Aujol. A variational approach to removing multiplicative noise. SIAM J. Appl. Math., 68(4):925-946, 2008.

[4] D. Auroux. From restoration by topological gradient to medical image segmentation via an asymptotic expansion. Math. Comput. Model., 49(11):2191-2205, 2009.

[5] D. Auroux, L. Jaafar Belaid, and B. Rjaibi. Application of the topological gradient method to tomography. In ARIMA Proc. TAMTAM’09, 13:91-104, 2010.

[6] D. Auroux and M. Masmoudi. Image processing by topological asymptotic expansion. J. Math. Imaging Vis., 33(2):122-134, 2009.

[7] E. Beretta, M.-C Cerutti, A. Manzoni, and D. Pierotti. An asymptotic formula for boundary potential perturbations in a semilinear elliptic equation related to cardiac electrophysiology. Math. Models Methods Appl. Sci., 26(4):645-670, 2016.

[8] J. Céa, S. Garreau, Ph. Guillaume, and M. Masmoudi. The shape and topological optimizations connection. Comput. Methods Appl. Mech. Engrg., 188(4):713-726, 2000.

[9] G. Chen and J. Zhou. Boundary Element Methods with Applications to Nonlinear Problems, volume 7 of Atlantis Studies in Mathematics for Engineering and Science. Springer Science \& Business Media, Amsterdam-Paris, 2010.

[10] H.-D. Cheng, J. Shan, W. Ju, Y. Guo, and L. Zhang. Automated breast cancer detection and classification using ultrasound images: A survey. Pattern recognition, 43(1):299-317, 2010.

[11] D. Colton and R. Kress. Inverse Acoustic and Electromagnetic Scattering Theory, volume 93 of Applied Mathematical Sciences. Springer Science \& Business Media, New York, 2012.

[12] G. Dong, Z. Guo, and B. Wu. A convex adaptive total variation model based on the gray level indicator for multiplicative noise removal. Abstract and Applied Analysis, 2013(Article ID 912373), 2013.

[13] A. Drogoul and G. Aubert. The topological gradient method for semi-linear problems and application to edge detection and noise removal. Inverse Problem and Imaging, 10(1):51-86, 2016.

[14] A. Drogoul. Topological gradient method applied to the detection of edges and fine structures in imaging. PhD thesis, Université Nice Sophia Antipolis, October 2014.

[15] V. S. Frost, J. A. Stiles, K. S. Shanmugan, and J. C. Holtzman. A model for radar images and its application to adaptive digital filtering of multiplicative noise. IEEE Transactions on Pattern Analysis and Machine Intelligence, (PAMI) 4(2):157-166, 1982.

[16] S. Garreau, P. Guillaume and M. Masmoudi. The topological asymptotic for PDE systems: The elasticity case. SIAM J. Control Optim., 39(6):1756-1778, 2000.

[17] M. Iguernane, S.-A. Nazarov, J.-R. Roche, J. Sokolowski, and K. Szulci. Topological derivatives for semilinear elliptic equations. Int. J. Appl. Math. Comput. Sci., 19(4-5):191-205, 2009.

[18] L. Jaafar Belaid, M. Jaoua, M. Masmoudi, and M.-L. Siala. Application of the topological gradient to image restoration and edge detection. Engineering Analysis with Boundary Elements Journal, 32(11):891-899, 2008.

[19] Z. Jin and X. Yang. Analysis of a new variational model for multiplicative noise removal. J. Math. Anal. Appl., 362(2):415-426, 2010 .

[20] Z. Jin and X. Yang. A variational model to remove the multiplicative noise in ultrasound images. J. Math. Imaging Vis., 39(1):6274,2011

[21] K. Krissian, R. Kikinis, C-F. Westin, and K.G. Vosburgh. Speckle-constrained filtering of ultrasound images. In 2005 IEEE Computer Society Conference on Computer Vision and Pattern Recognition (CVPR'05), volume 2, pages 547-552, 2005. 
[22] D. Kuan, A. Sawchuk, T. Strand, and P. Chavel. Adaptive restoration of images with speckle. IEEE Transactions on Acoustics, Speech, and Signal Processing, 35(3):373-383, 1987.

[23] A. Lopes, E. Nezry, R. Touzi, and H. Laur. Structure detection and statistical adaptive speckle filtering in sar images. International Journal of Remote Sensing, 14(9):1735-1758, 1993.

[24] T. Loupas. Digital image processing for noise reduction in medical ultrasonicss. PhD thesis, University of Edinburgh, UK, 1988.

[25] J.-C. Nédélec. Acoustic and Electromagnetic Equations: Integral Representations for Harmonic Problems. Springer-Verlag, New York, 2001.

[26] L. Rudin, P.-L. Lions, and S. Osher. Multiplicative denoising and deblurring: theory and algorithms. In S. Osher and N. Paragios, editors, Geometric Level Sets in Imaging, Vision and Graphics, pages 103-119. Springer, Berlin, 2003.

[27] J. Tasinkevych and I. Trots. Circular Radon transform inversion technique in synthetic aperture ultrasound imaging: an ultrasound phantom evaluation. Archives of Acoustics, 39(4):569-582, 2015.

[28] A. Theljani, Z. Belhachmi, M. Kallel, and M. Moakher. Multiscale fourth-order model for image inpainting and low-dimensional sets recovery. Mathematical Methods in the Applied Sciences, 40(10):3637-3650, 2017.

[29] V. Thomée and L. Wahlbin. On Galerkin methods in semilinear parabolic problems. SIAM J. Numer. Anal., 12(3):378-389, 1975.

[30] Z. Wang, A.-C. Bovik, H.-R. Sheikh, and E. P. Simoncelli. Image quality assessment: From error visibility to structural similarity. IEEE Transactions on Image Processing, 13(4):600-612, 2004.

[31] M.-F. Wheeler. A priori $L_{2}$ error estimates for Galerkin approximations to parabolic partial differential equations. SIAM J. Numer. Anal., 10(4):723-759, 1973. 